\title{
Spatial Assessment of Heat Exposure in Bay Cities: A Case of Xiamen in China
}

\author{
Haifeng Yang ${ }^{1,2, *}$, Ya'nan $\mathrm{Wang}^{3}$, and Wei Shui ${ }^{1,2,3}$ \\ ${ }^{1}$ Fujian Spatial Information Research Center, 350116 Fuzhou, China \\ ${ }^{2}$ Key Laboratory of Spatial Data Mining \& Information Sharing, Ministry of Education, 350116 Fuzhou, China \\ ${ }^{3}$ College of Environment and Resources, Fuzhou University, 350116 Fuzhou, China
}

\begin{abstract}
With the global warming, the rapid urbanization process and the increasing intensity and scope of human activities, extreme high temperature and cooresponding high temperature events were frequent, meanwhile, the degree of urban exposure to high temperatures was growing. The research constructed the heat exposure evaluation index system by taking the heat exposure evaluation index system in Xiamen, a gulf-based city with high-temperature. This study evaluated the influence factors, spatial differentiation features and hotspots of the heat exposure based on the methods of geo-spatial analysis, emergy accounting theory, expert consultation and analytic hierarchy process in Xiamen. The research results were expected to promote the theoretical development of urban heat exposure researches, and to provide decision-making reference for heat exposure assessment, regulation and adaptation in Xiamen and similar high temperature cities.
\end{abstract}

\section{Introduction}

In recent decades, due to the effects of global warming and urban heat island effects, heat disasters around the world have occurred frequently, causing a large number of casualties and economic losses ${ }^{[1]}$. According to the fifth report of the Intergovernmental Panel on Climate Change (IPCC), the frequency of heat waves in Europe, Australia, Asia and other regions was increasing. For example, in the summer of 2003 , the heat weather in France caused more than 14,000 death ${ }^{[2]}$. At the same time, Italy, Portugal, the Netherlands and other countries also recorded thousands of deaths due to heat disaster. As a significant mold of natural disasters in urban region, its intensity and frequency of occurrence and duration will be significantly enhanced in the future. Therefore, heat disasters have become one of the hot-spots in the research of urban natural disasters.

At present, the research on heat disasters is mainly centralized on the occurrence characteristics of heat disasters, the temporal and spatial distribution, the degree of impact of heat disasters and the warning system of heat disasters. More attention was paid to the dangers and vulnerabilities of past heat events, instead of the study of exposure. Heat exposure reflects the extent to which a complex human system suffered disasters or the degree of risk, depending on the probability that human and region exposure to hazardous events ${ }^{[3]}$. In the selection of heat exposure assessment indicators, the effects of temperature and population density factors were normally considered. For example, Jones et al.(2015) used SRES A2 climate scenario data to study the exposure of the future population to extreme high temperatures in the United States ${ }^{[4]}$; Aubrecht et al.(2013) used the spatio-temporal variation of high temperature days to analyze the characteristics of heat exposure areas in Washington ${ }^{[5]}$. However, in fact, under different factors such as humidity and wind speed, the human body feels differently about the same temperature ${ }^{[6]}$. The heat exposure was not only influenced by temperature and population density factors, because of the high air humidity and abundant wind resources in the bay cities, the effects of air humidity and wind on heat exposure also needs to be considered. Therefore, our research explored the spatial characteristics of heat exposure in bay cities by integrating multi-climate and population factors, in order to provide targeted decision support for humans to cope with future high temperature risks.

\section{Study area}

Xiamen City was affiliated to Fujian Province, located at $117^{\circ} 53^{\prime} 36^{\prime \prime}-118^{\circ} 25^{\prime} 17^{\prime \prime} \mathrm{E}, 24^{\circ} 25^{\prime} 02^{\prime \prime}-24^{\circ} 54^{\prime} 37^{\prime \prime} \mathrm{N}$, under the jurisdiction of Siming, Huli, Jimei, Haitang, Tong'an and Xiang'an six municipal districts, which were vital central cities, ports and scenic tourist cities in the southeast coast (Fig.1). They belong to the subtropical maritime monsoon climate with an average annual temperature of $21^{\circ} \mathrm{C}$. Its land area was over $1,690 \mathrm{~km}^{2}$, the territorial waters was over $390 \mathrm{~km}^{2}$, and the resident population was about 4.01 million in 2017.

\footnotetext{
* Corresponding author:yanghaifengtun@163.com
} 


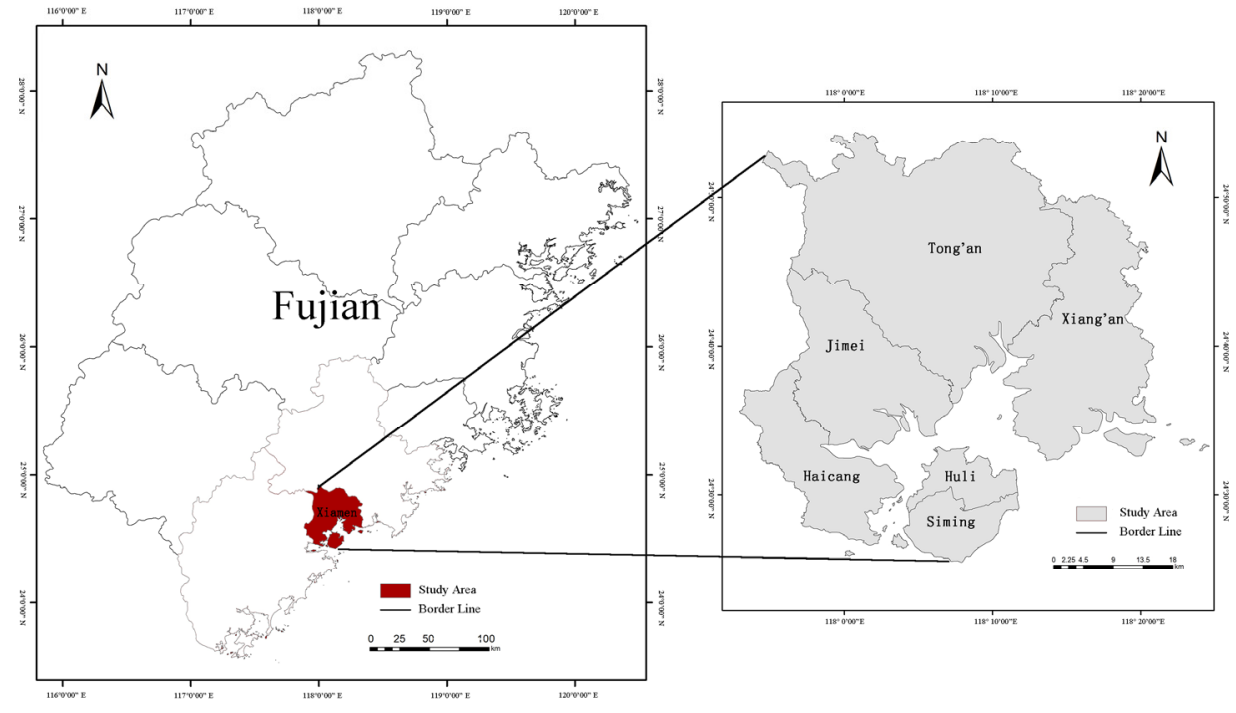

Fig.1 Location of Xiamen City in Fujian Province

Xiamen municipality was one of the earliest special economic zones established in China. It has caused a significant heat island effect in the process of rapid urbanization. According to meteorological statistics, there were 26 high-temperature heat waves during 1974 to 2014 , the average temperature per 10 years rising was $0.23^{\circ} \mathrm{C}$, and the extreme temperature was up to $39.2^{\circ} \mathrm{C}$. By the end of the century, if we do not consider the ecological environment, energy conservation, emission reduction and other factors, continue to take the extensive development routes, the annual average temperature of Xiamen will rise by $6.8^{\circ} \mathrm{C}$, but an increase of temperature that modern human can tolerate is $2^{\circ} \mathrm{C}$, which will seriously affect the production and daily life of residents and bring about a series of ecological and environmental problems.

\section{Data and methodology}

\subsection{Framework of heat exposure indicators}

On the basis of the traditional heat exposure index framework, combined with the characteristics of the ecoenvironment of the bay city, the Xiamen heat risk assessment index system (Table 1) was established. Heat exposure mainly selects the indicators of surface temperature, population density, humidity and wind energy. Among them, temperature has been empirically tested in the health effects of high temperature disasters. Remote sensing images were used to invert the surface brightness temperature to represent the surface temperature under cloudless sky to obtain refined surface temperature and ground thermal field variation. The population density reflected the exposure of the population in the region during high temperature disasters. The large population concentration in urban areas and high social stratification means that some urban resident had a particularly high vulnerability to climate change; coastal metropolis had higher air humidity. Meanwhile the wind resources were abundant, the temperature only represents the degree of cold and warm air that cannot fully express the human body's feeling of cold and warm to the environment. Under the same temperature conditions, people will also have different feelings of cold and warm due to different air humidity and wind speed.

\subsection{Data preprocessing}

\subsubsection{Surface temperature inversion}

By downloading the Landsat8 remote sensing satellite images with the highest temperature of $35 \mathrm{C}$, with no signal noise from July to September in 2015, and selected the TIRS10 band, which was with strong stability. The surface temperature inversion was performed based on the atmospheric correction method. The specific formulas were as follows:

$$
\begin{gathered}
B\left(T_{S}\right)=\left[L_{\lambda}-L \uparrow-\tau(1-\varepsilon) L \downarrow\right] / \varepsilon \tau \\
T_{\mathrm{s}}=K_{2} / \ln \left(K_{1} / \mathrm{B}\left(T_{\mathrm{s}}\right)+1\right)
\end{gathered}
$$

\begin{tabular}{|c|c|c|c|c|c|}
\hline $\begin{array}{l}\text { Criteria } \\
\text { layer }\end{array}$ & $\begin{array}{l}\text { Indicator } \\
\text { layer }\end{array}$ & $\begin{array}{c}\text { Data } \\
\text { Sources }\end{array}$ & $\begin{array}{c}\text { Spatial } \\
\text { resolution }\end{array}$ & $\begin{array}{c}\text { Indicator } \\
\text { pretreatment }\end{array}$ & $\begin{array}{l}\text { Index } \\
\text { weight }\end{array}$ \\
\hline \multirow{4}{*}{ Exposure } & Surface temperature & Remote sense image & $30 \mathrm{~m}$ & Surface temperature inversion & 0.6107 \\
\hline & Humidity & Meteorological data & - & Kriging interpolation & 0.1123 \\
\hline & Wind energy & $\begin{array}{l}\text { Land cover and Meteorological } \\
\text { data }\end{array}$ & $30 \mathrm{~m}$ & Wind emergy account & 0.0670 \\
\hline & Population density & Multisource geographic data & $100 \mathrm{~m}$ & Population density estimate & 0.2097 \\
\hline
\end{tabular}

Table 1. Indicator system of heat risk assessment in Xiamen 
Where $\mathrm{B}\left(\mathrm{T}_{S}\right)$ was the blackbody thermal radiance according to Planck's law, $\mathrm{T}_{S}$ was the true temperature of the surface; $L_{\lambda}$ was the thermal infrared radiation brightness value received by the satellite sensor, $\mathrm{L} \uparrow$ and $\mathrm{L} \downarrow$ represented the atmospheric upward radiance and atmospheric downward radiation Luminance respectively, $\tau$ was the transmittance of the atmosphere in the thermal infrared band, $\varepsilon$ was the surface specific emissivity; $\mathrm{K}_{1}$ and $\mathrm{K}_{2}$ were the thermal infrared calibration constants, where $\left.\mathrm{K}_{1}=774.89 \mathrm{~W} \mathrm{~m}^{-2} \mathrm{sr}^{-1} \mu \mathrm{m}^{-1}\right), \mathrm{K}_{2}=1321.08 \mathrm{~K}$.

\subsubsection{Emergy map of wind kinetic energy}

Emergy analysis was a relatively accurate environmental accounting method and GIS can provide visualize spatial information, and an emergy-GIS framework was employed in this study ${ }^{[7]}$. Wind energy was the kinetic energy generated by air flow. The spatial distribution of wind energy in Xiamen was obtained by using the data of the weather station from July to September in 2015 to characterize the effect of wind on the mitigation of high temperature. The specific formula was as follows:

$$
E_{W}=A \times \rho \times C_{D} \times v^{3} \times t \times T_{R}
$$

where $\rho$ was the air density $\left(1.3 \mathrm{~kg} / \mathrm{m}^{3}\right), \mathrm{C}_{\mathrm{D}}$ was the drag coefficient (Bare land: 0.01; grassland, cultivated land, shrubs: 0.02; woodland: 0.03), $v$ was the wind speed, and $\mathrm{T}_{\mathrm{R}}$ was the emergy transformation ratio of the wind kinetic energy $\left(2.45 \times 10^{3} \mathrm{sej} / \mathrm{j}\right)$.

\subsubsection{Population density estimate}

The production of the population density spatial datasets in 2015 principally follows the methodologies outlined in Stevens et al (2015), a new semi-automated dasymetric modeling approach had been built that incorporates census and a wide range of open access ancillary datasets in a flexible extent, "Random Forest" estimation technique ${ }^{[8]}$. A combination of widely available, remote sensor and geospatial datasets (eg. settlement locations, settlement extents, land cover, roads, building maps, health facility locations, satellite nightlights, vegetation, topography and refugee camps) contribute to the modeled dasymetric weights and then the Random Forest model is used to generate a gridded prediction of population density at $100 \mathrm{~m}$ spatial resolution. This prediction layer was then used as the weight surface to perform dasymetric redistribution of census counts at a country level.

\subsection{Index weight and evaluation model}

In our research, the index weights were calculated by the Delphi method and the analytic hierarchy process(AHP). The main steps were as follows: 1) Design the comparison scale between the heat exposure indicators after consulting the expert opinions; 2) To judge and compare the importance of the indicators, construct a hierarchical structure model, and obtain the quantifiable factor ranking results; 3) Obtain the comparison judgment matrix, perform the consistency test of the judgment matrix and calculate the weight of each evaluation standard, and the consistency test result shows $\mathrm{CR}=0.003<0.1$, which meet the consistency requirements. Therefore, the determined index weight results were reliable (Table 1).

The data was processed by the standard deviation method (Equation 1); the heat exposure index was obtained by superimposing the index weights and the standardized values (Equation 2).

$$
\begin{aligned}
& \mathrm{X}_{\mathrm{i}}^{*}=\left\{\begin{array}{l}
\frac{X_{i}-X_{i, \min }}{X_{i, \max }-X_{i, \min }}(\text { Positive }- \text { index }) \\
\frac{X_{i, \max }-X_{i}}{X_{i, \max }-X_{i, \min }}(\text { Inverse }- \text { index })
\end{array}\right. \\
& \mathrm{V}=\sum_{i=1}^{n} w_{i} \times X_{i}^{*}
\end{aligned}
$$

Where $\mathrm{X}_{\mathrm{i}}^{*}$ was the normalized value of the first indicator; $X_{i}$ was the actual value of the first indicator; $\mathrm{X}_{\mathrm{i}, \max }$ and $\mathrm{X}_{\mathrm{i}, \min }$ were the maximum and minimum values of the indicator respectively; $\mathrm{V}$ was the heat exposure index; $\mathrm{W}_{\mathrm{i}}$ was the weight of the first indicator; $\mathrm{n}$ was the number of indicators.

\section{Results}

\subsection{Spatial characteristics of various indicators}

The spatial distribution of the influence coefficient of each index was obtained by using the standardized formula. There was significant spatial difference between the surface temperature, wind energy, humidity and population density (Fig.2), which was the main influence factor of high temperature exposure in Xiamen.

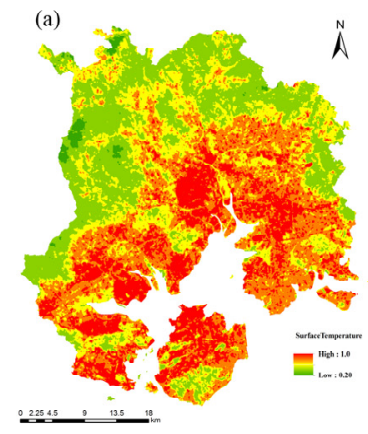

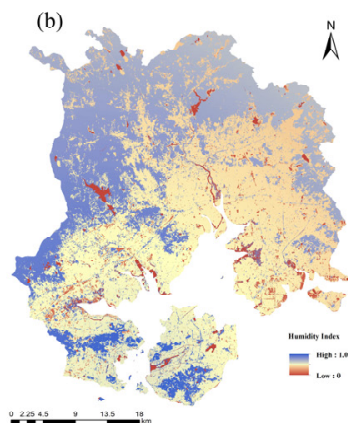

Fig.2 Spatial distribution of influence coefficient
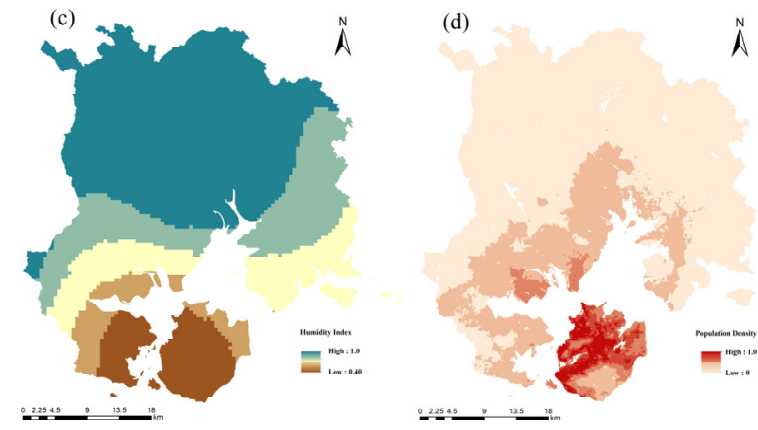
Table 2. The heat exposure area statistics Xiamen unit:( $\left.\mathrm{km}^{2} ; \%\right)$

\begin{tabular}{|c|c|c|c|c|c|c|c|c|c|c|c|c|}
\hline \multirow{2}{*}{ Levers } & \multicolumn{2}{|c|}{$\begin{array}{c}\text { Huli } \\
\text { District }\end{array}$} & \multicolumn{2}{|c|}{$\begin{array}{l}\text { Siming } \\
\text { District }\end{array}$} & \multicolumn{2}{|c|}{$\begin{array}{c}\text { Jimei } \\
\text { District }\end{array}$} & \multicolumn{2}{|c|}{$\begin{array}{l}\text { Haicang } \\
\text { District }\end{array}$} & \multicolumn{2}{|c|}{$\begin{array}{l}\text { Tong'an } \\
\text { District }\end{array}$} & \multicolumn{2}{|c|}{$\begin{array}{c}\text { Xiang'an } \\
\text { District }\end{array}$} \\
\hline & Area & $\begin{array}{c}\text { Propor } \\
\text { Tion }\end{array}$ & Area & $\begin{array}{c}\text { Propor } \\
\text { tion }\end{array}$ & Area & $\begin{array}{c}\text { Propor } \\
\text { tion }\end{array}$ & Area & $\begin{array}{c}\text { Propor } \\
\text { tion }\end{array}$ & Area & $\begin{array}{l}\text { Propor } \\
\text { tion }\end{array}$ & Area & $\begin{array}{c}\text { Propor } \\
\text { tion }\end{array}$ \\
\hline Low & 0 & 0 & 0 & 0 & 2.20 & 0.95 & 0.64 & 0.43 & 14.37 & 2.29 & 0.17 & 0.04 \\
\hline Sub-low & 0 & 0 & 0 & 0 & 76.18 & 32.83 & 32.11 & 21.78 & 346.39 & 55.22 & 73.16 & 19.24 \\
\hline Medium & 0.20 & 0.40 & 13.51 & 20.45 & 51.49 & 22.19 & 37.79 & 25.64 & 186.10 & 29.67 & 106.18 & 27.93 \\
\hline Sub-high & 5.97 & 11.92 & 19.73 & 29.86 & 87.58 & 37.75 & 71.42 & 48.44 & 79.96 & 12.75 & 127.17 & 33.45 \\
\hline High & 43.90 & 87.68 & 32.83 & 49.69 & 14.57 & 6.28 & 5.44 & 3.69 & 0.42 & 0.07 & 0.48 & 0.13 \\
\hline
\end{tabular}

\subsection{Heat exposure spatial characteristics}

Used the natural fracture method, the heat exposure index data was divided into low, sub-low, medium, subhigh, and high grades (Figure 3). The heat exposure of Xiamen city has a spatial distribution characteristic of "from south to north raise like ladders", that is, the heat exposure in the northern and western parts of the urban periphery is low, general, and the heat exposure in the southern part of the Xiamen is higher.The low-exposure and sub-low exposure areas were concentrated in the urban fringe area, which was mainly covered by the mountain and water; and the medium exposure area was the transitional area between the low exposure and the high exposure, mainly covered by mountain, water and urban construction, this regional was mainly used for village construction, and its wide distribute but scattered;The sub high exposure areas were mainly distributed in the urban center area,which was located in the north of the coastline. This regional was mainly serviced as urban residential land and urban infrastructure land; the high exposure area presents the characteristics of "aggregate distribution", from east to west, but mainly in Xiamen Island and urban new areas, which was located in the south of the coastline, the degree of heat exposure was gradually increased, from north to south.

The average exposures of Huli, Siming, Jimei, Haicang, Tong'an and Xiang'an were 0.97, 0.86, 0.63, $0.67,0.51$ and 0.64 , respectively, meanwhile the proportion of high exposure areas was $87.68 \% .49 .69 \%$, $6.28 \%, 3.69 \%, 0.07 \%$ and $0.13 \%$ (Table 2). Among them, the highest exposure average values and the highest proportion of exposure area was Huli District, while the area of Siming and Huli exposure areas accounted for 0 in the exposure of low and sub-low. Compared with other areas, that was significantly different.

\subsection{Heat exposure hot spots analysis}

In order to deep explain the spatial correlation degree of heat exposure in Xiamen, the global Moran's I and G estimates are calculated. The value of Global Moran's I was 0.33 , accord with the assumption of normal distribution, the $\mathrm{Z}$ value of the I index test was 134.62, indicating heat exposure had a significant positive spatial auto correlation, presenting a very strong spatial agglomeration state.

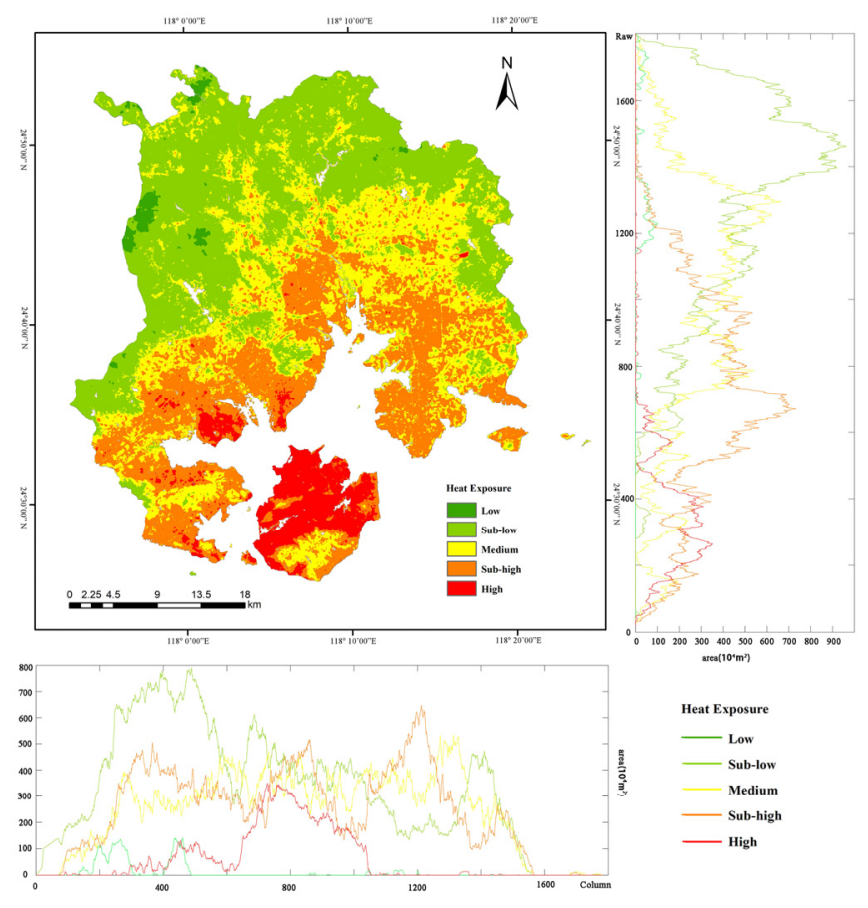

Fig.3 Spatial distribution of heat exposure in Xiamen 


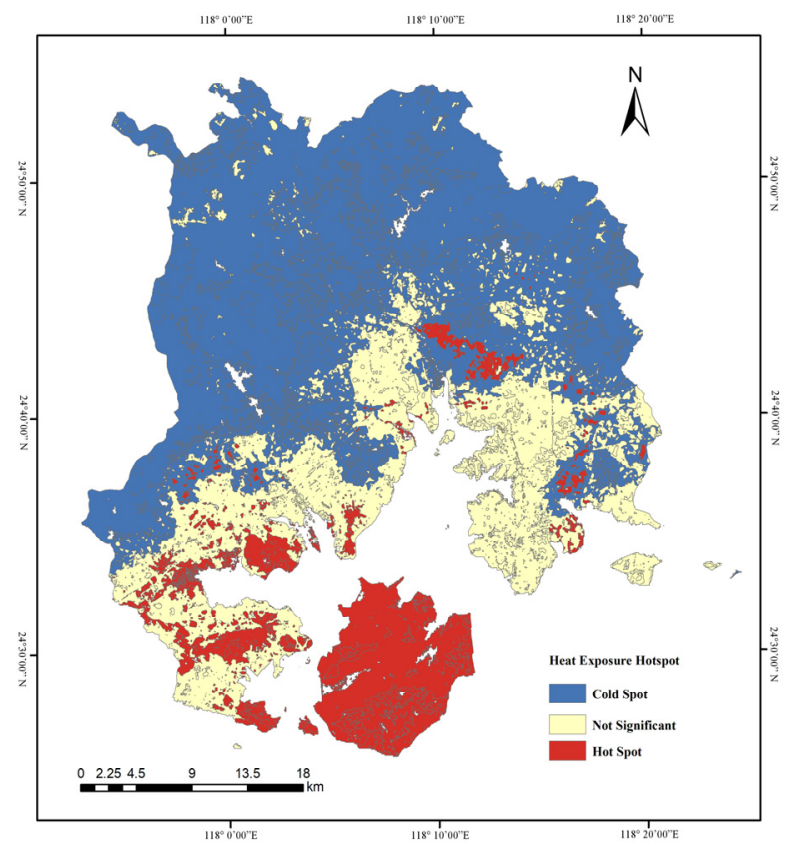

Fig.4 Hot spot analysis of heat exposure in Xiamen

The global Moran's I and G estimates can indicate whether the high temperature exposure was spatially agglomerated,and its global features, but it often mask local instability in some extent, while the spatial hotspot - analysis method (Getis-OrdGi*), accordance to measure parts area characteristics, and test whether there were statistically significant high and low values, which was the spatial distribution of hot and cold spots. The statistical significance of the reliability was $95 \%$, and the hot spot analysis of heat exposure in Xiamen was carried out (Fig.4). Heat exposed hot-spots were mainly distributed in Siming and Huli districts, and a few were distributed in industrial entrepreneurship parks and urban rural integration zones of Haicang and Tong'an District; heat exposure cold spots were concentrated in the peripheral areas of Tong'an, Xiang'an and Jimei District, mainly due to the characteristics of population evacuation, low surface temperature and abundant wind energy resources; The mildly exposed area was between the hot and the cold spot area, which played a role of transitional relaxation.

\section{Discussion and Conclusions}

This paper evaluated the influence factors, spatial differentiation characteristics and hot-spots of heat exposure, taken the high temperature municipality of Xiamen as an example.This research was different from the conventional demographic density and temperature indicators, which was selected by the predecessors for heat exposure, and used geo-spatial analysis technology to inverse the surface temperature and estimation the population density, which can more accurately assess urban the heat exposure characteristics, and fully considered the high air humidity and the ecological environment characteristics of rich in wind energy resources in the bay-type high-temperature city, the evaluation results were more scientific and reasonable, and can provide a reference for decision-making for the government to implement high-temperature prevention and control work.

Based on the distribution and influence factors of heat exposure in Xiamen, it was recommended that government departments of the same type of heat cities should focus on how to reduce heat exposure in urban centers, such as strengthen high-temperature prevention projects and increase the community's attention and services for the high-risk groups, which was sensitive to high temperatures. And, focused on high exposure areas such as industrial areas and urban-rural integration, formulate urban government's adaptive planning for high-temperature, developed decision support systems, and improved the city's high-temperature adaptability and cope capacity.

\section{References}

1. R. Rasch, Environ. Urban. 28, 1 (2015)

2. M. Poumadère, M. Le, Risk Anal 25, 6 (2010)

3. P. Xie, Y. Wang, Y. Liu, J. Peng, Acta Geogr. Sin. 70, 7 (2015)

4. B. Jones, B. O'Neill, L. McDaniel, Nat. Clim. Chag. 5, 7 (2015)

5. C Aubrecht, D Özceylan, Envir.Inter. 56, 1 (2013)

6. C Yagtou, ASHVE Trans. 53, 1 (1947)

7. C Wang, S Zhang, W Yan,R. Wang, J. Liu, Y. Wang, Sci. Rep. 6, (2016)

8. F.Stevens, A. Gaughan, C. Linard, A. Tatem, Plos One 10, 2 (2015) 